\title{
Efeitos do Tai Chi Chuan na incidência de quedas, no medo de cair e no equilíbrio em idosos: uma revisão sistemática de ensaios clínicos aleatorizados
}

\author{
The effects of Tai Chi Chuan in occurrences of falls, fear of falling and \\ balance in the elderly: a systematic review of randomized control trials
}

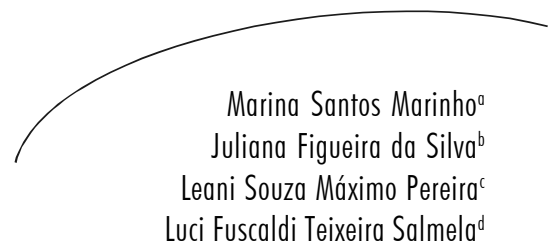

Marina Santos Marinho $0^{\circ}$ Juliana Figueira da Silva Luci Fuscaldi Teixeira Salmela

\section{Resumo}

O processo de envelhecimento é um dos fatores que, através da deterioração do equilíbrio, influenciam o aumento do número de quedas e resultam em sérias complicações, como o medo de cair. O Tai Chi Chuan é uma arte marcial milenar oriental considerada um exercício de intensidade leve a moderada. A prática do Tai Chi Chuan na população idosa apresenta efeitos positivos no controle do equilíbrio, na incidência de quedas e no medo de cair. Sendo assim, o objetivo deste estudo foi realizar uma revisão sistemática que evidenciasse o efeito do Tai Chi Chuan na incidência de quedas, no medo de cair e no equilíbrio em idosos. Foram pesquisados ensaios clínicos aleatórios nas bases de dados Medline, Lilacs, Cochrane e PEDro. Dois revisores avaliaram a qualidade dos estudos e realizaram a extração dos dados de forma independente. Foram selecionados seis artigos, que abrangeram 816 participantes. Uma análise dos resultados demonstrou uma resposta positiva do Tai Chi Chuan na redução da incidência de quedas e no medo de cair em idosos sedentários e que vivem na comunidade. $\mathrm{O}$ mesmo efeito não foi observado em relação à melhora da estabilidade postural.

\section{Abstract}

The aging process is one of the factors that influence the increasing number of falls and result in complications like fear of falling. The Tai Chi Chuan (TCC) is an oriental martial art considered as light to moderate intensity exercise. The
Palavras-chave: envelhecimento; acidentes por quedas; medo; tai ji; terapia por exercicio; equilibrio musculosqueletico

Correspondência / Correspondence

Marina Santos Marinho

R. Frederico Cornélio, 111

130770-050, Belo Horizonte, MG, Brasil

E-mail: marinasantosm@yahoo.com.br 
practice of TCC has positive effects on balance, occurrence of falls and fear of falling on the elderly. This study aimed to, through a systematic review, investigate the effects of TCC in risk of falls, fear of falling and balance in the elderly. Randomized controlled trials were investigated on databases Medline, Lilacs, Cochrane and PEDro. Two reviewers analyzed the quality of the studies and extract data independently. Six studies were selected, covering 816 participants. The results demonstrated a positive effect of TCC on decreasing occurrences of falls and fear of falling in sedentary community-dwelling elderly. The same effect was not observed for the postural stability.
Key words: aging; accidental falls; fear; Tai Ji; exercise therapy; musculoskeletal equilibrium

\section{INTRODUÇ̃̃̃O}

O processo de envelhecimento fisiológico vem acompanhado de várias mudanças em nível funcional, sensorial (visão, sistema vestibular e propriocepção) e motor (força, coordenação e resistência muscular), que interferem no sistema do controle postural ou do equilibrio. ${ }^{6}$

A deterioração do equilíbrio com o processo de envelhecimento é um dos fatores que influenciam o aumento do número de quedas, o que resulta em sérias complicações. ${ }^{13}$ As quedas em idosos são causas relevantes de fratura de quadril, resultando em mais de 250.000 fraturas anuais. ${ }^{2}$ Estudos realizados no Brasil constataram grande número de internações decorrentes de quedas, as quais, em geral, apresentaram custo-dia e gasto médio elevado para o sistema de saúde. ${ }^{18}$

Apresentar uma ou mais quedas é um fator de risco independente para desenvolver o medo de cair. ${ }^{8,10} \mathrm{~A}$ incidência e prevalência do medo de cair na população idosa é significativamente grande, gerando um forte impacto na função e na qualidade de vida dos idosos. ${ }^{8}$
A queda pode não ser uma causa direta de morte, mas seu efeito cumulativo e comorbidades associadas indicam um aumento na morbidade e mortalidade e um declínio funcional. ${ }^{26}$

O sedentarismo no envelhecimento é fator que contribui para uma deterioração adicional do controle postural; ${ }^{22}$ já os exercícios físicos constituem uma terapêutica para minimizar as alterações fisiológicas do processo de envelhecimento, sendo importantes para a promoção da saúde e qualidade de vida nos idosos. ${ }^{5}$

Idosos fisicamente ativos apresentam uma melhora do equilíbrio, se comparados a idosos sedentários. Isto sugere que os exercícios físicos têm sido usados como importante intervenção para prevenção e redução de quedas na população idosa. ${ }^{22.4,12,20}$

O Tai Chi Chuan (TCC) é uma arte marcial milenar oriental voltada para maior conscientização corporal, sendo considerado um exercício de intensidade leve a moderada, e tem sido pesquisado recentemente pela cultura ocidental. ${ }^{14} \mathrm{~A}$ prática do TCC na população idosa apresenta efeitos favo- 
ráveis no controle do equilíbrio, flexibilidade, no sistema cardiorespiratório e no aspecto psicossocial. . $^{14,9,28}$

A melhora no controle postural e no equilíbrio, secundária ao TCC, deve-se principalmente ao aumento e manutenção da força muscular dos músculos posturais dos membros inferiores ${ }^{37} \mathrm{e}$ à reeducação do equilíbrio, através do treino de desestabilização do centro de massa. ${ }^{7}$ O TCC também proporciona uma reação muscular mais rápida frente a desequilíbrios posturais ${ }^{38}$ e melhora a propriocepção de tornozelo e joelho. ${ }^{30,39}$

Wolf et al. ${ }^{32}$ identificaram sete elementos terapêuticos na prática do TCC que promovem melhora na postura, no equilíbrio e na marcha dos idosos. Os elementos terapêuticos encontrados foram movimentos lentos $\mathrm{e}$ contínuos, que são realizados com flexão progressiva dos joelhos e com o aumento gradual da amplitude de movimento. Os exercícios são realizados com cabeça e tronco alinhados e com $70 \%$ de peso corporal em uma perna e posteriormente a transferência para a outra, trabalhando o suporte de peso unilateral e equilíbrio. Os movimentos são simétricos e diagonais, tanto em membros superiores quanto inferiores, e ocorrem em círculos direcionados e sincronizados com os movimentos dos olhos (trabalhando fortes componentes rotacionais da visão e labirinto).

Wolfson et al. ${ }^{36}$ realizaram estudo com treino de equilibrio e fortalecimento muscular dos membros inferiores em 110 idosos residentes na comunidade durante três meses. Os idosos foram alocados em quatro grupos: con- trole, treino de equilíbrio, treino de força muscular e treinos de equilíbrio associados à força muscular. Após seis meses dessas intervenções, os idosos participantes do estudo receberam um programa de intervenção de TCC, por seis meses. O objetivo foi verificar se o TCC conservaria a mesma performance adquirida no final do programa de exercícios de equilíbrio e fortalecimento muscular. Os autores concluíram que o TCC foi responsável pela manutenção dos ganhos em equilíbrio e força muscular adquiridos pelas intervenções citadas anteriormente. ${ }^{36}$

Várias pesquisas indicaram que a prática do TCC constitui uma intervenção eficaz no equilíbrio de idosos. ${ }^{28,37,7,38,30,39,17,29}$ Conseqüentemente, encontra-se uma diminuição no número de quedas e no medo de cair em idosos praticantes do TCC. . $^{15,25,31,24}$

Sendo assim, o objetivo deste estudo foi o de realizar uma revisão sistemática que evidencie o efeito do TCC na prevenção ou diminuição do risco de quedas, no medo de cair e na melhora do equilíbrio em idosos.

\section{METODOLOGIA}

\section{Estratégia de busca}

Os estudos foram identificados a partir das bases de dados Lilacs, Medline, Cochrane e PEDro, limitando o período de publicação entre 1995 a 2005, nos idiomas português ou inglês. Foram usadas como palavras-chave Tai Chi Chuan, idosos (elderly), quedas (falls), medo 
de queda (fear of falling) e equilíbrio (balance). Durante a busca houve o cruzamento dessas palavras com Tai Chi Chuan.

\section{Seleção dos Estudos}

Foram considerados somente ensaios clínicos aleatórios que utilizaram o TCC como intervenção em indivíduos idosos com idade maior ou igual a 65 anos para prevenção de quedas, diminuição do medo de cair e melhora do equilíbrio. Para que um ensaio clínico fosse incluído, este deveria conter um grupo experimental e um grupo controle.

Os artigos que obedeceram aos critérios de inclusão foram analisados independentemente por dois revisores (S.J.F., M.S.M.), que os classificaram como "incluído", "excluído" ou "duvidoso". O índice Kappa foi utilizado para quantificar o nível de concordância entre os avaliadores. Nos casos em que houve divergência, os itens discrepantes foram revistos e discutidos até a obtenção de consenso sobre a pontuação.

\section{Avaliação metodológica}

A metodologia dos estudos incluídos foi avaliada através da escala PEDro, ${ }^{16}$ baseada na lista de Delphi. ${ }^{27}$ A escala PEDro é constituída de 11 itens que avaliam a qualidade metodológica dos ensaios clínicos aleatórios, observando dois aspectos do estudo: se ele apresenta validade interna e se contém informações estatísticas suficientes para torná-lo interpretável. ${ }^{16}$ A escala não avalia a validade externa, significância, ou o tamanho do efeito do tratamento. ${ }^{21} \mathrm{~A}$ escala PEDro tem apresentado confiabilidade "razoável" a "boa". ${ }^{16}$ Os estudos foram qualificados através do mesmo instrumento de forma independente por dois revisores (M.S.M., S.J.F.). O índice Kappa foi utilizado para quantificar o nível de concordância entre os avaliadores. Nos casos em que houve divergência, os itens discrepantes foram revistos e discutidos até a obtenção de consenso sobre a pontuação.

Estudos com escore igual ou maior a 5 $(50 \%)$ foram considerados de alta qualidade, de acordo com a literatura pesquisada. ${ }^{19}$

\section{Extração dos dados dos artigos}

As informações contidas nos artigos selecionados por esta revisão foram resumidas, de forma independente, nos seguintes tópicos: autor/ano; caracterização da amostra; intervenção com TCC; intervenção do grupo controle e resultados encontrados. Foram extraídos, de cada estudo, os seguintes parâmetros: duração da prática do TCC (semanas), intensidade (horas/minutos) e freqüência (número de vezes por semana) da intervenção.

\section{RESULTADOS}

\section{Estudos Identificados}

A pesquisa inicial, realizada nas bases de dados eletrônicas, identificou 122 artigos. Após análise do título e leitura dos resumos, conforme os critérios de inclusão predeterminados, 20 artigos foram selecionados. Destes, após 
análise pela escala de classificação de PEDro (tabela 1), foram escolhidos, para participarem da revisão sistemática, seis artigos que abrangeram 816 participantes. Os artigos excluídos foram os que não se adequaram ao tema pro- posto, não apresentaram grupo controle, revisões da literatura, intervenção em população não-idosa e intervenção de TCC com outras finalidades (condicionamento cardiorrespiratório, artrite reumatóide, dentre outros).

Tabela 1 - Classificação metodológica avaliada pela escala PEDro

\begin{tabular}{|c|c|c|c|c|c|c|}
\hline & $\begin{array}{l}\text { Wolf et al. } \\
(1996)^{31}\end{array}$ & $\begin{array}{c}\text { Wolf et al. } \\
(1997)^{33}\end{array}$ & $\begin{array}{l}\text { Wolf et al. } \\
(2003)^{35}\end{array}$ & $\begin{array}{l}\text { Li et al. } \\
(2004)^{15}\end{array}$ & $\begin{array}{l}\text { Sattin et al. } \\
(2005)^{24}\end{array}$ & $\begin{array}{l}\text { Zhang et al. } \\
\text { (2005) } 40\end{array}$ \\
\hline $\begin{array}{l}\text { 1- Critérios de inclusão } \\
\text { especificados* }\end{array}$ & $\mathrm{v}$ & $\mathrm{V}$ & $\mathrm{v}$ & $\mathrm{v}$ & $\mathrm{v}$ & $\mathrm{v}$ \\
\hline 2- Alocação aleatória & $\mathrm{v}$ & $\mathrm{v}$ & $\mathrm{v}$ & $\mathrm{v}$ & $\mathrm{v}$ & $\mathrm{v}$ \\
\hline $\begin{array}{l}\text { 3- Seqüência de alocação } \\
\text { oculta }\end{array}$ & $\mathrm{x}$ & $\mathrm{x}$ & $\mathrm{x}$ & $\mathrm{x}$ & $\mathrm{v}$ & $\mathrm{x}$ \\
\hline $\begin{array}{l}\text { 4- Igualdade entre grupos } \\
\text { no pré-tratamento }\end{array}$ & $\mathrm{v}$ & $\mathrm{v}$ & $\mathrm{v}$ & $\mathrm{v}$ & $\mathrm{v}$ & $\mathrm{v}$ \\
\hline 5- Participantes cegos & $\mathrm{x}$ & $\mathrm{x}$ & $x$ & $x$ & $\mathrm{x}$ & $\mathrm{x}$ \\
\hline 6- Terapeutas cegos & $\mathrm{x}$ & $\mathrm{x}$ & $\mathrm{x}$ & $\mathrm{x}$ & $\mathrm{v}$ & $\mathrm{x}$ \\
\hline 7- Examinadores cegos & $\mathrm{v}$ & $\mathrm{x}$ & $\mathrm{v}$ & $\mathrm{x}$ & $\mathrm{v}$ & $\mathrm{x}$ \\
\hline $\begin{array}{l}\text { 8- Follow-up de pelo } \\
\text { menos } 85 \% \text { dos } \\
\text { participantes }\end{array}$ & $\mathrm{v}$ & $\mathrm{v}$ & $\mathrm{v}$ & $\mathrm{x}$ & $\mathrm{x}$ & $\mathrm{v}$ \\
\hline $\begin{array}{l}\text { 9- Análise por "intenção- } \\
\text { de-tratar" }\end{array}$ & $\mathrm{x}$ & $\mathrm{x}$ & $\mathrm{v}$ & $\mathrm{v}$ & $\mathrm{v}$ & $\mathrm{x}$ \\
\hline $\begin{array}{l}\text { 10- Comparações } \\
\text { estatísticas entre grupos }\end{array}$ & $\mathrm{v}$ & $\mathrm{v}$ & $\mathrm{v}$ & $\mathrm{v}$ & $\mathrm{v}$ & $\mathrm{v}$ \\
\hline $\begin{array}{l}11 \text { - Especificado o } \\
\text { tamanho do efeito e } \\
\text { variabilidade }\end{array}$ & $\mathrm{v}$ & $\mathrm{v}$ & $\mathrm{v}$ & $\mathrm{v}$ & $\mathrm{v}$ & $\mathrm{v}$ \\
\hline Total (em 10 pontos) & 6 & 5 & 7 & 5 & 8 & 5 \\
\hline
\end{tabular}

Legenda: v - sim; x - não. Obs: * a pontuação do primeiro item (critérios de inclusão especificados), por ser referente à validade externa, não é considerada no escore final (MOSELEY ${ }^{19}$ et al., 2002).

\section{Características gerais dos artigos selecionados}

A maioria dos estudos incluídos nesta revisão detalha informações sobre o método de randomização. Quanto às variáveis pesqui- sadas, os estudos relatam que: a duração da prática do TCC (semanas) variou entre oito e 48 semanas, a intensidade (horas/minutos) entre 45 a 90 minutos e a freqüência (número de vezes por semana) de duas a sete vezes por semana, conforme o Quadro 1. 
Quadro 1 - Características dos artigos selecionados nesta revisão sistemática

\begin{tabular}{|c|c|c|c|}
\hline Autor/Ano & Amostra & Intervenções & Resultados e Conclusões \\
\hline $\begin{array}{l}\text { Wolf et al. } \\
(1996)^{31}\end{array}$ & $\begin{array}{l}\text { Idosos que } \\
\text { vivem na } \\
\text { comunidade, } \\
\text { hígidos, com } \\
\text { média de } \\
\text { idade de } 76,2 \\
\text { anos }(\mathrm{n}=200)\end{array}$ & $\begin{array}{l}\text { Intervenção Tai Chi Chuan } \\
\text { Tai Chi Chuan ( } \mathrm{n}=72): 2 \mathrm{x} / \text { semana; } 45 \\
\text { minutos, durante } 15 \text { semanas. } \\
\text { Intervenção grupo controle } \\
\text {-Treino de Equilíbrio } \\
\text { Computadorizado ( } \mathrm{n}=64) \text { : } \\
\text { 1x/semana; } 45 \text { minutos, durante } 15 \\
\text { semanas. } \\
\text {-Controle -educacional ( } \mathrm{n}=64) \text { : } \\
\text { 1x/semana,durante } 15 \text { semanas }\end{array}$ & $\begin{array}{l}\text { Resultados } \\
\text { Houve redução do medo de cair no grupo } \\
\text { Tai Chi Chuan }(\mathrm{P}=0,46) \text { em comparação } \\
\text { com o Grupo Controle }(\mathrm{P}=0,58) \text {. } \\
\text { Ocorreu uma redução no risco de queda } \\
\text { de } 47,5 \% \text { no grupo do Tai Chi Chuan } \\
\text { Conclusão } \\
\text { A intervenção com Tai Chi Chuan teve } \\
\text { impacto favorável nos índices de } \\
\text { fragilidade e na redução da ocorrência de } \\
\text { quedas. }\end{array}$ \\
\hline $\begin{array}{l}\text { Wolf et al. } \\
(1997)^{33}\end{array}$ & $\begin{array}{l}\text { Idosos que } \\
\text { vivem na } \\
\text { comunidade, } \\
\text { hígidos, com } \\
\text { média de } \\
\text { idade de } 70 \\
\text { anos }(\mathrm{n}=72)\end{array}$ & $\begin{array}{l}\text { Intervenção Tai Chi Chuan } \\
\text { Tai Chi Chuan }(\mathrm{n}=24) 2 \mathrm{x} / \text { semana,1 } \\
\text { hora, durante } 15 \text { semanas } \\
\text { Intervenção grupo controle } \\
\text {-Treino de Equilíbrio } \\
\text { computadorizado ( } \mathrm{n}=24) \text { : } 1 \mathrm{x} / \text { semana, } \\
1 \text { hora, durante } 15 \text { semanas. } \\
\text { - Controle- Educacional }(\mathrm{n}=24) \text { : } \\
1 \mathrm{x} / \text { semana, } 1 \text { hora, durante } 15 \\
\text { semanas. }\end{array}$ & $\begin{array}{l}\text { Resultados } \\
\text { Treino de equilíbrio computadorizado } \\
\text { revelou uma melhora da estabilidade, mas } \\
\text { poucas mudanças na estabilidade postural } \\
\text { foi observada no grupo Tai Chi Chuan e } \\
\text { no grupo controle educacional. Os } \\
\text { sujeitos do grupo Tai Chi Chuan } \\
\text { apresentaram menor medo de cair } \\
\text { comparado aos demais grupos. } \\
\text { Conclusão } \\
\text { Houve redução no número de quedas, } \\
\text { porém este efeito não parece estar } \\
\text { associado à melhora da estabilidade } \\
\text { postural, mas com a maior confiança dos } \\
\text { idosos. }\end{array}$ \\
\hline $\begin{array}{l}\text { Wolf et al. } \\
(2003)^{35}\end{array}$ & $\begin{array}{l}\text { Idosos que } \\
\text { vivem na } \\
\text { comunidade, } \\
\text { em transição } \\
\text { para a } \\
\text { fragilidade, } \\
\text { com média } \\
\text { de idade de } \\
81 \text { anos } \\
(\mathrm{n}=286)\end{array}$ & $\begin{array}{l}\text { Intervenção Tai Chi Chuan } \\
\text { Tai Chi Chuan (n=145) } 2 \mathrm{x} / \text { semana, } \\
\text { com progressão de } 60 \text { a } 90 \text { minutos, } \\
\text { durante } 48 \text { semanas. } \\
\text { Intervenção grupo controle } \\
\text { Grupo Controle (Educacional) } \\
\text { (n=141) } 1 \mathrm{x} / \text { semana } \\
1 \text { hora, durante } 48 \text { semanas. }\end{array}$ & $\begin{array}{l}\text { Resultados } \\
\text { Não houve diferença estatisticamente } \\
\text { significativa no risco de quedas entre o } \\
\text { Grupo Tai Chi Chuan e o Grupo } \\
\text { Controle, porém, a porcentagem de } \\
\text { participantes que caiu durante as } 48 \\
\text { semanas de intervenção foi menor no } \\
\text { Grupo Tai Chi Chuan que no Grupo } \\
\text { Controle (P=0,13 } \\
\text { Conclusão } \\
\text { O Tai Chi Chuan não reduz a incidência } \\
\text { de quedas em idosos transicionais para a } \\
\text { fragiliadade, mas a direção dos efeitos } \\
\text { observadas neste estudo, juntamente com } \\
\text { os achados positivos na população idosa } \\
\text { hígida sugere que o Tai Chi Chuan tenha } \\
\text { uma importância clínica na redução de } \\
\text { quedas. }\end{array}$ \\
\hline
\end{tabular}


Quadro 1 - Características dos artigos selecionados nesta revisão sistemática (continuação)

\begin{tabular}{|c|c|c|c|}
\hline $\begin{array}{l}\text { Sattin et al. } \\
(2005)^{24}\end{array}$ & $\begin{array}{l}\text { Idosos que } \\
\text { vivem na } \\
\text { comunidade } \\
\text { em transição } \\
\text { para } \\
\text { fragilidade } \\
(\mathrm{n}=392)\end{array}$ & $\begin{array}{l}\text { Intervenção Tai Chi Chuan } \\
\text { Tai Chi Chuan ( } \mathrm{n}=158) \text { : } 2 \mathrm{x} / \text { semana, } \\
\text { com progressão de } 60 \text { a } 90 \text { minutos, } \\
\text { durante } 48 \text { semanas. } \\
\text { Intervenção grupo controle } \\
\text { Controle- Educacional ( } \mathrm{n}=153) \text { : } \\
\text { 1x/semana, 1hora, durante } 48 \\
\text { semanas. }\end{array}$ & $\begin{array}{l}\text { Resultados } \\
\text { Após o ajuste das variáveis, os achados do } \\
\text { ABC após } 12 \text { meses de intervenção foram } \\
\text { significativamente maiores no grupo Tai } \\
\text { Chi Chuan em relação ao grupo de } \\
\text { educação para o bem estar, sendo que } \\
\text { estas diferenças aumentaram com o } \\
\text { tempo (diferença aos } 12 \text { meses }=9.5 \\
\text { pontos, } 95 \% \text { de intervalo de confiança }= \\
\text { 4.8-14.2, P }<.001 \text { ) } \\
\text { Conclusão } \\
\text { A prática do Tai Chi Chuan causa um } \\
\text { aumento significativo na redução do } \\
\text { medo de cair em relação ao grupo } \\
\text { controle, nos idosos em transição para a } \\
\text { fragilidade. }\end{array}$ \\
\hline $\begin{array}{l}\text { Zhang et al. } \\
(2005)^{40}\end{array}$ & $\begin{array}{l}\text { Idosos } \\
\text { pouco } \\
\text { hígidos } \\
\text { (diminuição } \\
\text { relativa do } \\
\text { equilíbrio), } \\
\text { com idade } \\
\text { acima de } 60 \\
\text { anos }(\mathrm{n}=49 \text { ) }\end{array}$ & $\begin{array}{l}\text { Intervenção Tai Chi Chuan } \\
\text { Tai Chi Chuan }(\mathrm{n}=25): 7 \mathrm{x} / \text { semana; } 60 \\
\text { minutos, durante } 8 \text { semanas. } \\
\text { Intervenção grupo controle } \\
\text { Grupo Controle ( } \mathrm{n}=24) \text { : qualquer } \\
\text { outra atividade física intensa, pelo } \\
\text { menos } 3 \mathrm{x} / \text { semana; } 30 \text { minutos no } \\
\text { mínimo, durante } 8 \text { semanas }\end{array}$ & $\begin{array}{l}\text { Resultados } \\
\text { O grupo TCC apresentou efeitos } \\
\text { positivos no equilíbrio, ( } 25.7 \text { vs } 16.1, \mathrm{P}< \\
0.001) \text { na flexibilidade }(6.2 \text { vs } 3.0, \mathrm{P} \\
<0.001) \text { e na redução do medo de cair } \\
\text { (78.3 vs } 75.3, \mathrm{P}=0.006) \text { quando } \\
\text { comparado ao grupo controle após a } \\
\text { intervenção. }\end{array}$ \\
\hline & & & $\begin{array}{l}\text { Conclusão } \\
\text { Os resultados sugerem que com uma alta } \\
\text { freqüência e curta duração um programa } \\
\text { de treino de TCC pode melhorar o } \\
\text { equilíbrio, a flexibilidade e aumentar a } \\
\text { confiança dos idosos pouco hígidos. }\end{array}$ \\
\hline
\end{tabular}

Quanto à população total pesquisada, houve predomínio de idosos hígidos que viviam na comunidade, do sexo feminino, da cor branca e inativos. A presença de uma amostra homogênea, ou seja, com igualdade entre grupos no pré-tratamento, foi perceptível em todos os artigos selecionados. Nenhuma pesquisa selecionada apresentou o "cegamento" dos participantes do estudo.

\section{Qualidade metodológica}

A concordância entre os examinadores que avaliaram individualmente a qualidade da evidência utilizando a escala PEDro foi susbstancial ${ }^{1}$ com Índice $\mathrm{Ka}$ ppa de 0.79. Discordâncias foram observadas nos itens 3 e $8^{31}$ e 8 e $9^{35}$ da escala utilizada. (Tabela 1). 


\section{Características dos estudos selecionados}

As características dos estudos selecionados estão presentes no Quadro 1.

O ensaio clínico randomizado, da Atlanta FICSIT Grous, ${ }^{31}$ apresentou como objetivo avaliar o efeito de duas intervenções, o TCC e o treino de equilíbrio computadorizado, nas medidas primárias (indicadores de fragilidade biomédicos, funcionais e psicossociais) e medidas secundárias (ocorrência de quedas). Duzentos idosos que viviam na comunidade, com idade média de 76,2 anos, participaram do estudo. Eles foram alocados em três grupos: Grupo 1 - TCC, grupo 2 - treino de equilíbrio computadorizado e grupo 3 - controle - programa de educação para o bemestar. A intervenção durou 15 semanas, com as medidas primárias mensuradas antes e após a intervenção e com quatro meses de followup. A ocorrência de quedas foi monitorada continuamente durante o estudo. ${ }^{31}$

As medidas primárias constaram de indicadores biomédicos que incluíam força muscular (teste muscular de Nicholas MMT 01160 e a força muscular de preensão manual pela Jammar Smedley-type hand dynamometer), flexibilidade muscular (amplitude de movimento dos membros inferiores), composição corporal (medida da prega cutânea da parede abdominal anterior, peito e coxa) e resistência cardiovascular (tempo requerido para completar 12 minutos de caminhada e a medida da freqüência cardíaca e pressão arterial antes e após essa caminhada). A segunda medida foi o tempo de ocorrência de quedas (FICSIT Falls and Injury Committe).
Como resultado, encontrou-se um declínio da força muscular de preensão manual em todos os grupos pesquisados. Houve diminuição do medo de cair no grupo de idosos que praticou o TCC, comparado ao grupo controle $(\mathrm{p}=0,046$ e $\mathrm{p}=0,058$, respectivamente). Uma redução na incidência de múltiplas quedas de $47,5 \%$ também foi verificada no grupo que praticou o TCC. Os autores concluíram que uma intervenção moderada de TCC tem impactos favoráveis nos índices de fragilidade biomédicos e psicossociais, além do efeito positivo sobre a diminuição da ocorrência de quedas nos idosos. ${ }^{31}$

O mesmo grupo de pesquisadores Atlanta FICSIT Group, ${ }^{33}$ citados anteriormente, realizaram outro estudo com 72 idosos retirados da mesma amostra, com os mesmos parâmetros de intensidade e duração do tratamento e randomizados nos mesmos três grupos citados: grupo1 - TCC, grupo 2 - treino de equilíbrio computadorizado e grupo 3 - controle- programa de educação para o bem-estar. No entanto, o foco do estudo desta vez foi verificar os efeitos da prática do TCC na estabilidade postural. Os sujeitos foram avaliados pelo Chattecx Balance System ${ }^{T M}$. Os resultados obtidos foram que as medidas de equilibrio revelaram grande estabilidade após intervenção dos sujeitos do grupo 2 , mas pequena mudança na estabilidade dos sujeitos dos grupos 1 e 3 . O grupo 1 reduziu o medo de cair (odds ratio $=0.298, \mathrm{p}=0.08$ ) comparado aos grupos 2 e 3 (odds ratio $=1.436$, e 1.436, respectivamente; $\mathrm{P}=0.13)$. Os autores concluíram que, ao contrário do treino computadorizado, o TCC não melhorou as medidas de estabilidade postural..$^{33}$ 
O ensaio clínico randomizado realizado por Wolf et al. ${ }^{35}$, com 48 semanas de duração, teve como objetivo determinar se um programa intensivo de TCC (grupo 1) seria melhor para reduzir o risco de cair, se comparado ao grupo controle (grupo 2) com um programa de educação para o bem-estar. A amostra foi constituída por idosos com predisposição a quedas e apresentando critérios de fragilidade transicional. Trezentos e onze idosos, entre 70 a 97 anos, não-institucionalizados, participaram do estudo. Dados demográficos, tempo de ocorrência até a primeira queda e todas as quedas subseqüentes, medidas funcionais (Sickness Impact Profile, Perfil de Impacto de Doença, Center for Epidemiologic Studies, Depression Scale, Escala do Centro de Estudos Epidemiológicos da Depressão), uso de medicamentos, nível de atividade física, comorbidades e aderência à intervenção foram medidos a cada quatro meses durante um ano. A taxa de risco (RR) de cair não foi estatisticamente diferente no grupo 1 em relação ao grupo 2, grupo controle $(\mathrm{RR}=0.75,95 \%$ de intervalo de confiança $(\mathrm{CI})=0.52-1.08), \mathrm{P}=0.13)$. Após as 48 semanas de intervenção, $46 \%(n=132)$ dos participantes não relataram quedas; a percentagem de participantes que caiu foi $47,6 \%$ do grupo 1 e $60,3 \%$ para o grupo 2 . Com base nos dados citados, o estudo concluiu que a prática intensa do TCC não reduziu o risco de cair em idosos em transição para a fragilidade. ${ }^{35}$

Li et al. ${ }^{15}$ realizaram um estudo randomizado, com o objetivo de verificar se existe melhora no equilíbrio funcional com a inter- venção do TCC e se essa prática está relacionada com subseqüentes reduções nas quedas em idosos. Duzentos e cinqüenta e seis idosos hígidos, inativos, que viviam na comunidade, com idade média de 77,48 anos, participaram do estudo. Os participantes foram alocados da seguinte maneira: grupo 1 - intervenção através do TCC ou no grupo 2 - grupo controle, de exercícios de fortalecimento muscular. A intervenção durou seis meses (com as medidas sendo realizadas no pré-tratamento, com três meses de intervenção, pósintervenção e em um follow-up de seis meses). As medidas de equilíbrio funcional foram mensuradas pela Escala de Equilíbrio de Berg (BBS), índice de marcha dinâmico (DGI) e alcance funcional (Funcional Reach - FR), e a medida de quedas, através da contagem de quedas, na qual cada participante as anotava em calendário diário de quedas. Os resultados obtidos foram que o TCC apresentou efeito positivo nas medidas de equilíbrio funcional após a intervenção (odds ratio (OR), $0.27,95 \%$ de intervalo de confiança, 0.07 $0.96, p=0.04$ para a escala de Berg; OR, $0.27,95 \%$ de intervalo de confiança, 0.09 $0.87, p=0.03$ para DGI e OR, $0.20,95 \%$ de intervalo de confiança, $0.05-0.82, \mathrm{p}=$ 0.03 para o alcance funcional). O grupo de idosos submetidos à prática do TCC apresentou também redução significativa na incidência de quedas $(\mathrm{p}<0.001)$ nos seis meses pós-intervenção, quando comparado ao grupo controle. A partir destes dados, os autores concluíram que a melhora no equilíbrio funcional, decorrente da prática do TCC, está associada a uma subseqüente redução na incidência de quedas nos idosos. ${ }^{15}$ 
Um estudo controlado randomizado foi realizado por Sattin et al. ${ }^{24}$, com a mesma amostra, mesmo processo de randomização e os mesmos parâmetros de intensidade e duração do tratamento do estudo de Wolf et al.. ${ }^{35}$ O objetivo desse estudo foi determinar se um programa de prática intensa de TCC seria melhor para reduzir o medo de quedas, quando comparado a um programa de educação para o bem-estar, em idosos com predisposição a quedas e apresentando critérios de fragilidade. Trezentos e onze idosos, entre 70 a 97 anos, não-institucionalizados, foram alocados em dois grupos, grupo 1, treino de TCC, e grupo 2, grupo controle, programa de educação para o bem-estar. A duração do estudo foi de 48 semanas. Duas escalas (Activities-specific Balance Confidence Scale (ABC) e Fall Efficacy Scale) foram aplicadas a cada quatro meses durante um ano, para mensurar a média de quedas em atividades requeridas e a confiança dos idosos em realizar atividades funcionais diárias. Dados demográficos, tempo até a primeira queda e todas as quedas subseqüentes, medidas funcionais, escala do centro de estudos epidemiológicos da depressão, uso de medicamentos, nível de atividade física, comorbidades e aderência à intervenção também foram quantificados. A escala utilizada $\mathrm{ABC}$ apresentou resultados similares quando aplicada na primeira avaliação entre ambos os grupos, demonstrando a homogeneidade da amostra pesquisada. A partir do oitavo mês, o grupo que praticou o TCC apresentou redução significativa do medo de cair, quando comparado ao controle $(57.9$ vs 49.0, $\mathrm{P}<001)$ e no final do estudo (59.2 vs 47.9, $\mathrm{P}<001)$. Após 12 meses de interven- ção com o TCC, os resultados apresentados pelos idosos foram significativamente maiores em relação ao grupo controle. É importante ressaltar que esses valores se tornaram mais significativos com o aumento do tempo da prática de TCC pelos idosos $(12$ meses $=$ 9.5 pontos, $95 \%$ de intervalo de confiança $=$ 4.8-14.2, $\mathrm{P}<001)$. A partir disso, o estudo conclui que a prática intensa do TCC resulta em aumento significativo na redução do medo de cair em relação ao grupo controle, nos idosos em transição para a fragilidade..$^{24}$

ZHANG et al. ${ }^{40}$ realizaram estudo com o objetivo de investigar o efeito de um programa intenso de TCC durante oito semanas, na função fisiológica e no medo de cair, em idosos com diminuição relativa do equilíbrio. Quarenta e nove idosos com idade de 60 anos ou mais foram randomizados em dois grupos. No grupo 1, a intervenção terapêutica foi o TCC, e o grupo 2 controle, idosos que realizavam qualquer outra atividade física intensa, pelo menos 3 vezes por semana, durante no mínimo 30 minutos. Medidas de performance física, incluindo apoio unipodálico, flexão de tronco e velocidade da marcha, foram realizadas.

O instrumento utilizado para mensurar o medo de cair e a confiança na realização das atividades funcionais diárias foi o Falls Efficacy Scale (FES). O grupo de idosos que praticou o TCC apresentou efeitos positivos no equilíbrio, (25.7 vs 16.1, P < 0.001), na flexibilidade (6.2 vs $3.0, \mathrm{P}<0.001)$ e na redução do medo de cair (78.3 vs 75.3, $\mathrm{P}=0.006$ ), quando comparado ao grupo controle. Os resul- 
tados sugerem que, com uma alta freqüência e curta duração, um programa de treino de TCC pode melhorar o equilíbrio, a flexibilidade e aumentar a confiança em idosos, com diminuição relativa do equilíbrio. Isto sugere a eficiência do treino de TCC como um programa capaz de prevenir quedas em populações idosas de alto risco. ${ }^{40}$

\section{DISCUSSÃO}

O resultado desta revisão sistemática mostrou que o TCC apresenta resposta positiva na redução da incidência de quedas e do medo de cair. Entretanto, não foi possível observar o mesmo efeito em relação à melhora da estabilidade postural. Observa-se que há uma limitação de ensaios clínicos aleatorizados, representando esse tipo de intervenção, nos parâmetros queda, medo de cair e estabilidade postural.

Ganhos na redução da incidência de quedas e no medo de cair em idosos, adquiridos com a prática do TCC, são mantidos por alguns meses após a intervenção, e no caso dos estudos selecionados para esta revisão, a manutenção variou de dois a 12 meses. Isso sugere que o TCC foi responsável pela manutenção desses ganhos em longo prazo, como comprovado anteriormente pelo estudo de Wolfson et al.. ${ }^{36}$

$\mathrm{Na}$ análise descritiva da população total, observa-se certa homogeneidade das amostras dos estudos selecionados para esta revisão, uma vez que todos utilizaram idosos que vivem na comunidade e inativos, o que per- mite uma conclusão específica para essa amostra. A diferenciação encontrada entre as amostras dos estudos foi a presença de idosos hígidos e idosos em estágio de transição para a fragilidade, o que pode resultar num viés no resultado final. Wolf et al..$^{35}$ utilizaram uma amostra de idosos em transição para a fragilidade e concluíram que o TCC não reduziu a incidência de quedas nos participantes. Tal resultado poderia estar associado ao fato de uma amostra de idosos em transição para a fragilidade reduzir o poder do seu estudo, quando comparado a uma amostra de idosos hígidos, que, segundo o autor, já possui efeito positivo comprovado na redução da incidência de quedas. ${ }^{31}$

O quinto critério da escala PEDro ${ }^{16}$ exige que todos os participantes do experimento sejam incapazes de distinguir se receberam o tratamento ou não - ou seja, se foram "cegos". O TCC é uma atividade física com todas as suas implicações. O grupo controle recebeu apenas um programa educacional, e tanto o grupo controle quanto o grupo TCC eram submetidos a atividades em conjunto. Portanto, intervenções distintas em grupo, invariavelmente, impediram um "mascaramento" adequado dos participantes, por isso todos os estudos incluídos nesta revisão não pontuaram neste quesito. Além disso, alguns estudos demonstraram que o grupo controle tinha consciência da existência do grupo de intervenção, tornando ainda mais difícil esse cegamento. ${ }^{15,34}$

A análise dos parâmetros, duração da prática (semanas) e freqüência (número de vezes por semana) da intervenção do TCC utiliza- 
dos durante os estudos revelou falta de padronização. A duração da prática do TCC variou de oito ${ }^{40}$ a 48 semanas. ${ }^{24,35} \mathrm{~A}$ falta de padronização quanto à freqüência, duas ${ }^{15,24,31,33,35}$ a sete $^{40}$ vezes por semana também foi observado nos estudos analisados. Logo, a utilização de parâmetros distintos impossibilitou determinar quais destes são mais eficazes para a obtenção dos benefícios desejados.

Um aspecto bastante relatado nos estudos selecionados foi a continuidade da prática do TCC após a intervenção do estudo e dos meses de follow up. Foram encontrados períodos de até dois anos de realização do TCC após a conclusão do estudo. ${ }^{33}$ Retrata-se constantemente na literatura que o TCC encoraja essa população idosa a manter um estilo de vida ativo e incorporar essa atividade no seu dia-a-dia, podendo ser considerado, portanto, uma atividade terapêutica com boa adesão pela população idosa. ${ }^{3} \mathrm{O}$ TCC, quando comparado a treino de equilíbrio computadorizado e palestras educacionais, parece que promoveu uma maior adesão dos idosos a essa atividade. O TCC é uma atividade física com benefícios que vão além da melhora do equilíbrio funcional e redução na incidência de quedas, é prazeroso e promove a socialização. ${ }^{15}$

\section{CONCLUSÃO}

Através dos estudos analisados, concluiuse que o TCC apresenta uma resposta positiva ao reduzir a incidência de quedas e o medo de cair em idosos hígidos e sedentários que vivem na comunidade. Entretanto, são necessários ainda mais estudos do tipo ensaios clínicos "aleatorizados" para verificar os efeitos do TCC na redução da incidência de quedas, medo de cair e melhora do controle postural nos idosos em transição para a fragilidade.

\section{NOTAS}

Graduada em Fisioterapia pela Universidade Federal de Minas Gerais

R Frederico Cornélio, 111 - 130770-050, Belo Horizonte, MG, Brasil

E-mail: marinasantosm@yahoo.com.br

b Graduada em Fisioterapia pela Universidade Federal de Minas Gerais

R. Joaquim Pereira, 700 - 31565-370, Belo Horizonte, MG, Brasil

E-mail: jufigueira2003@yahoo.com.br

c,d Professoras Doutoras Adjuntas do Departamento de Fisioterapia, Universidade Federal de Minas Gerais Av. Antônio Carlos, 6627 - 31270-905, Belo Horizonte, MG, Brasil

Emails: leanismp.bh@zaz.com.br e 1fts@ufmg.br

\section{REFERÊNCIAS}

1. Abraira V. El índice kappa. Semergen 2000; 27(5): 247-9.

2. Braithwaite R S, Col N F, Wong J B. Estimating hip fracture morbidity, mortality and costs. J Am Geriatr Soc 2003; 51(3): 364-70.

3. Chen K, Snyder M, Krichbaum K. Facilitators and barriers to elders' practice of Tai Chi. J Holist Nurs 2001; 19(3): 238-55.

4. Day L, et al. Randomised factorial trial of falls prevention among older people living in their own homes. BMJ 2002; 325 (7356): 128-33.

5. Elley CR, et al. Effectiveness of counselling patients on physical activity in general practice: cluster randomized controlled trial. BMJ 2003; 326(73930): 793-8. 
6. Hageman PA, Leibowitz M, Blanke D. Age and gender effects on postural control measures. Arch Phys Med Rehabil 1995; 6( 10): 961-5.

7. Hass C, et al. The influence of Tai Chi training on the center of pressure trajectory during gait initiation in older adults. Arch Phys Med Rehabil 2004; 85(10): 1593-8.

8. Helen WL. Incidence and risk factors for developing fear of falling in older adults. Public Health Nutr 2005; 22: 45-52.

9. Hong Y, Li JX, Robinson PD. Balance control, flexibility and cardiorespiratory fitness among older Tai Chi practitioners. $\mathrm{Br}$ J Sports Med 2000; 34(1): 29-34.

10. Howland J, et al. Fear of falling among the community-dwelling elderly. J Aging Health 1993; 5(2): 229-43.

11. IBGE. Censo Demográfico, 2000. Rio de Janeiro: IBGE, 2001.

12. Judge JO. Balance training to maintain mobility and prevent disability. Am J Prev Med 2003; 25(3s): 150-6.

13. Konrad HR, Girardi M, Helfert R. Balance and aging. Laryingoscope1999; 109( 9): 1454-60.

14. Li JX, Hong Y, Chan KM. Tai Chi: physiological characteristics and beneficial effects on health. Br J Sports Med 2001; 35 ( 3): 148-56.

15. Li F, et al. Tai Chi: improving functional balance and predicting subsequent falls in older persons. Med Sci Sports Exerc 2004; 36(12): 2046-52.

16. Maher CG, et al. Reliability of the PEDro Scale for rating quality of randomized controlled trials. Phys Ther 2003; 83( 8): 713-21.

17. Mak MK, Ling P. Mediolateral sway in singleleg stance is the best discriminator of balance performance for Tai Chi practitioners. Arch. Phys. Med. Rehabil 2003: 84(5): 683-6.
18. Mello MP, Koisume MS, Gawryszewski V M. Mortes e internações por causas externas entre os idosos no Brasil: o desafio de integrar a saúde coletiva e atenção individual. Rev Assoc Med Bras 2004; 50(1): 97-103.

19. Moseley AM, et al. Evidence for physiotherapy practice: A survey of the Physiotherapy Evidence Database (PEDro). Australia Journal of Physiotherapy 2002; 48 ( 1): 43-9.

20. Nitz JC, Chey NL. The efficacy of a specific balance-strategy training programme for preventing falls among older people: a pilot randomized controlled trial. Age Ageing 2004; 33(1): 52-8.

21. PEDro. The Physiotherapy Evidence Database (PEDro) [acesso 2005 set 20].

Disponível em: URL: http:/ / www.pedro.fhs.usyd.edu.au

22. Perrin P, et al. Effects of physical and sporting activies on balance control in elderly people. Br J Sports Med 1999; 33(3):121-6.

23. Ramos LR, Veras RP, Kalache A.

Envelhecimento populacional: uma realidade brasileira. Rev Saúde Pública 1987; 21(3): 211-24.

24. Sattin RW, et al. Reducing in fear of falling through intense Tai Chi exercise training in older, transitionally frail adults. J Am Geriatr Soc 2005; 53( 7):1168-78.

25. Taggart HM. Effects of Tai Chi exercise on balance, functional mobility and fear of falling among older women. Applied Nursing Researh 2002; 15(4): 235-42.

26. Tinetti ME. Preventing falls in the elderly persons. The New England journal of medicine 2003; 348(18): 42-9.

27. Verhagen AP, et. al. The Delphy List: a criteria list for quality assessment of randomized clinical trials for conducting systematic reviews developed by Delphi Consensus. J Clin Epidemiol 1998; 51(12): 1235-41. 
28. Verhagen AP, et. al. The efficacy of Tai Chi Chuan in older adults: a systematic rewiew. Family practice 2004; 21(1): 107-13.

29. Willian WN, Tsang WY. Effect of 4- and 8wk intensive Tai Chi training on balance control in the elderly. Med Sci Sports Exerc 2004; 36( 4): 648-57.

30. Willian WN, Tsang WY. Effects of Tai Chi on joint proprioception and stability limits in elderly subjects. Med Sci Sports Exerc 2003; 35(12): 1962-71.

31. Wolf SL, et al. Reducing frailty and falls in older persons: an investigation of Tai Chi and computerized balance training. J Am Geriatr Soc 1996; 44(4): 489-97.

32. Wolf SL, Coogler C, Xu T. Exploring the basis for Tai Chi Chuan as a therapeutic exercise approach. Arch Phys Med Rehabil 1997; 78(8): 886-92.

33. Wolf SL, et al. The effect of Tai Chi Quan and computerized balance training on postural stability in older subjects. Phys Ther 1997; 77( 4): 371-81.

34. Wolf SL, et al. A study desing to investigate the effect of intense Tai Chi in reducing falls among older adults transitioning to frailty. Control Clin Trials 2001; 22( 6): 689-704.
35. Wolf SL, et al. Intense Tai Chi exercise training and fall occurrences in older, transitionally frail adults: a randomized controlled trial. J Am Geriatr Soc 2003; 51(12): 1693-701.

36. Wolfson L, et al. Balance and strength training in older adults: intervention gains and Tai Chi maintenance. J Am Geriatr Soc 1996; 44( 5): 498-506.

37. Wu G, Zhao F, Zhou X. Improvement of isokinetic knee extensor strengh and reduction of postural sway in the elderly from long-term Tai Chi exercise. Arch Phys Med Rehabil 2002; 83(10): 1364-9.

38. Xu D, Li J X, Hong Y. Effect of regular'Tai Chi and jogging exercise on neuromuscular reaction in older people. Age Ageing 2005; 34( 5): 439-43.

39. Xu D, et al. Effect of Tai Chi exercise on proprioception of ankle and knee joints in old people. Br J Sports Med 2003; 38(1): 50-4.

40. ZhangJG, et al. The effects of Tai Chi Chuan on physiological function and fear of falling in the less robust elderly: An intervention study for preventing falls. Arch Gerontol Geriatr 2005; 42:107-16. In press.

Recebido em: 19/3/2007

Aceito: 04/5/2007 